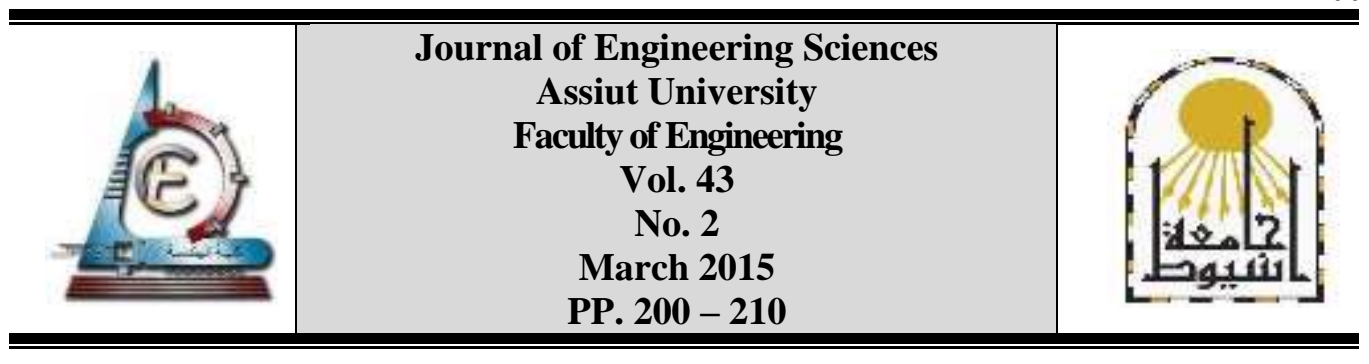

\title{
A LINEARIZATION TECHNIQUE FOR NONLINEAR SDOF SYSTEMS BASED ON BLOCK PULSE TRANSFORM
}

\author{
Hosein Ghaffarzadeh \\ Faculty of Civil Engineering, University of Tabriz, Tabriz, Iran
}

(Received 25 March 2015; Accepted 3 May 2015)

\begin{abstract}
This paper addresses a linearization technique for a single degree of freedom (SDOF) linearizable nonlinear system based on block pulse (BP) transform. The BP transform gives effective tools to simplify control and system problems. The main goal of this work is on using BP transform properties in process of linearization of nonlinear problems. It is necessary to compare the results obtained using this method with other traditional methods to verify the effectiveness of the proposed method. The efficiency of BP transform method compared with the traditional equivalent linearization (EL) method. Both numerical simulations are applied to the Duffing nonlinear oscillator system to demonstrate the feasibility of the proposed method based on BP transform. Finally, the results of the comparison between the approaches depicted the proposed method is able to approximate the nonlinear systems behavior. The results showed the superiority of the proposed approach in the sense that it is more accurate by computational advantageous.
\end{abstract}

Keywords: Linearization, block pulse transform, nonlinearity, Duffing oscillator

\section{Introduction}

Study of nonlinearity in dynamic systems has been a prominent area of research in last decades. This inherent phenomenon inevitably occurs in physical systems. In mechanical and structural systems nonlinearities can arise in various forms and usually becomes progressively more significant as the motion amplitude increases. The major sources of nonlinearity arise from misalignment, looseness, temperature effects, impedance mismatching, pre-load, exciter problems and overloads [1]. Nonlinear systems may show complicated behavior, such as limit cycles, bifurcations and even chaos which are difficult to predict. Besides in practical applications, due to the high intensity nature and often complex nature of non-stationary environmental loads such as wind loads, sea waves and earthquakes, the systems subjected to these loadings may experience excessive stress or displacements that results inelastic behavior [2]. To describe real observed processes in field of engineering, researchers often use mathematical models. For dynamic processes, these models contain many different types of equations such as ordinary or partial differential equations, difference equations, and algebraic equations. In general case, due to the nature of the considered problems are nonlinear, there is no exact solution for such equations. Except for some special cases, the solutions are approximate [3]. There are diverse approaches which have been developed over the years to treat the nonlinear problems. Most commonly used methods 
include Perturbation, Monte Carlo simulation and well known harmonic balance method which is one of the main techniques for obtaining approximate analytic solutions to nonlinear ordinary differential equations. Also there are the semi-analytical methods, such as the high order harmonic balance (HOHB) method which has been developed to avoid the tedious algebraic calculations involved in the classical harmonic balance method in processing the nonlinear term in the nonlinear dynamical system [4], the high dimensional harmonic balance (HDHB) method and the time domain collocation (TDC) method [5]. Meanwhile, among many methods dealing with a nonlinear system, linearization methods are the oldest and the most popular methods of approximation. To approximate the nonlinear problems a powerful linearization technique is required to analyze and predict nonlinear system's behavior in order to design an accurate and desirable scheme of a system during its operation under any excitations. One of the linearization methods is the Lyapunov linearization technique used to approximate a nonlinear system by a linear one that is around the equilibrium point, and it is expected the behavior of the linear system will be the same as that of nonlinear one in the equilibrium point [6]. The other one is feedback linearization method which is a common approach used in nonlinear control systems. This method is based on the idea of transforming original system model into equivalent linear one which is changed to the state variables and a suitable input instead [7,8]. Describing Function is another method for system linearization which is an extension of the frequency response method of linear control can be used as the approximate analysis to predict some important characteristics of nonlinear system including systems with hard nonlinearities [9]. Statistical equivalent linearization (EL) technique is commonly used approach in nonlinear problems. The statistical EL method is based on the idea that the nonlinear system is replaced by an equivalent linear equation by minimizing the difference between the two systems in some appropriate sense. In order to predict the response of this kind of system or to get an approximation solution of nonlinear equation this method is applied to estimate the accurate equivalent linear parameters. This method was proposed by Caughey [10] as a way to solve nonlinear vibration problems. Statistical EL method has proven to be very useful approximation technique in structural dynamics and earthquake engineering. All methods of statistical and EL can be considered in different fields such as state space, frequency domain, distribution space and characteristic functions space. Usually they consist of two main steps. In the first step, deal to find explicit or implicit analytical formulas for linearization coefficients based on the linearization criterion which is depend on unknown response characteristics such as mean value, variance, and higher-order moments. In the second step, deal to replace the unknown characteristics by the corresponding ones determined for linearized systems. It is worth mentioning that accuracy and feasibility of these solutions dependent on the type of nonlinearity and amplitude of external excitation forces. This method has been widely used [11,12]. Discrete-time (data sampled) systems have resulted in corresponding demand for designing and understanding these systems. These systems are governed by difference equations in which members are coupled to each other. One source of difference equations is the numerical evaluation of integrals. Also we could use the conventional Laplace transform to solve these difference equations [13].

Block pulse (BP) transform provides a useful tool to solve difference equations of any order with less computational costs. The BP transform originated from BP functions. The BP functions are a set of orthogonal functions with piecewise constant values and are usually applied as a useful tool in the analysis, synthesis, identification and other problems of control and systems science. This set of functions was first introduced to electrical 
engineers by Harmuth [14]. Some papers discussed the BP functions and their operational matrix for integration in order to reduce the complexity of expressions in solving certain control problems via Walsh functions. PurnachandraRao and RanganathaRao [15] used BP functions to determine the piecewise constant feedback controls for a finite linear optimal control problem of a power system. The proposed method is simple and has computationally advantageous. Sannuti showed that the application of BP functions results in an enormous reduction of computational effort over Walsh functions in control system applications [16]. In active control problem a new method proposed based on BP functions evolves minimizing computational costs of analytical approaches $[17,18]$.

The main objective of this study is the using of BP transform in linearization procedure through its easy and simple operation. The input - output relationships for a linearized system and nonlinear system are obtained using the BP transform. Following the basic procedure of the traditional EL approach, one can find the least mean square error between the linearized and nonlinear equations. The effectiveness of the proposed method is validated on nonlinear Duffing oscillator system. Different simulations used to verify the accuracy and feasibility of the proposed method, the traditional EL results of displacement have been compared with those obtained by this method. Results from this study presented that this method can approximate the nonlinear systems behavior for stationary excitation better than the traditional EL method.

Frequency response function (FRF) summarizes essential information to specify the dynamics of a structure. The FRF of linear and nonlinear system that is linearized by existing methods have been compared. This comparison confirmed the accuracy of proposed method.

The remaining of this paper is organized as follows. Section 2 presents the BP transform formulation. In section 3 linearization method based on BP transform is proposed. The simulations have been carried out to compare with the traditional EL method in Section 4 and followed by the conclusion in Section 5.

\section{Block pulse transform}

Block pulse (BP) transform originate form B pulse functions (BPFs). BP functions possess various properties such as disjointness, Orthogonality and completeness. These functions discretize time domain of a problem to a unit time interval. Discretization in time domain makes BP functions as a potential approximation method which has its accuracy and simplicity. Here we noted that BP transform may not provide better approximation in comparison with the linearization based on the EL approach. EL approach is also a rich and strong methodology. However, BP transform provides a simplified approximation method and its results can be compared with EL approach.

The block pulse transform method provides a technique for transforming a difference equation into an algebraic equation. It is very similar to z-transform method due to both methods can greatly facilitate the analysis, especially when we only desire responses at the sampling instants. But the block pulse transform defined with respect to the Laplace transform [19]. Consequently, the BP transform is the implementation of Black Pulse Functions in z-transform. The BP transform of function $f(t)$ is defined by:

$$
F(z)=\sum_{\mathbf{i}=0}^{\infty} f_{\mathbf{i}} z^{-\mathbf{i}}
$$


Where $f_{\mathrm{i}}$ are the coefficients of the terms $z^{-\mathbf{i}}(i=0,1,2, \ldots)$ in the power series which are the values of the sampled signal $f(t)$ at the corresponding time instants $h$ and $h$ is the sampling period. The summery of BP transform properties are following [20]:

For addition and subtraction of function $x(t)=f(t) \pm g(t)$, we have:

$X(z)=F(z) \pm G(z)$

For multiplied by a scalar $x(t)=k f(t)$, we have:

$X(z)=k F(z)$

For multiplication of function $x(t)=f(t) g(t)$, we have:

$$
X(z)=\sum_{\mathbf{i}=0}^{\infty} f_{\mathbf{i}} g_{\mathbf{i}} z^{-\mathbf{i}}
$$

For division of function $x(t)=f(t) / g(t)$ with $g(t) \neq 0$, we have:

$$
X(z)=\sum_{\mathbf{i}=0}^{\infty}\left(f_{\mathbf{i}} / g_{\mathbf{i}}\right) z^{-\mathbf{i}}
$$

and finally for derivative of function $x(t)=\frac{d f(t)}{d t}$, we have:

$$
X(z)=\frac{2}{h} \frac{1-z^{-1}}{1+z^{-1}} F(z)-f_{0}^{(0)} \frac{2}{h} \frac{1}{1+z^{-1}}
$$

where $f_{0}^{(0)}$ is the initial value of $f(t)$.

\section{Linearization based on BP transform}

We consider a SDOF nonlinear system subject to external signal $f(t)$ with the following motion equation:

$$
m \ddot{x}(t)+g(x, \dot{x})=f(t)
$$

where, $m$ is mass of system and $g(x, \dot{x})$ is the nonlinear function. Performing the BP transform both sides of (7) yields:

$$
m X_{1}(z)+G(z)=F(z)
$$

Where $X_{1}(z), G(z)$ and $F(z)$ are BP transform of $\ddot{x}(t), \quad g(x, \dot{x})$ and $f(t)$ respectively. Regarding to equation (8), the equation of an equivalent linearized system can be found as follows:

$$
m X_{1}(z)+c_{e q} X_{2}(z)+k_{e q} X(z)=F(z)
$$


In which, $c_{e q}$ and $k_{e q}$ are unknown damping and stiffness coefficients to be determined through linearization process. $X_{2}(z)$ and $X(z)$ are BP transform of $\dot{x}(t)$ and $x(t)$, respectively.

Replacement of a nonlinear system with an equivalent linear system will yield the error. The error, e, may be defined as:

$$
e=c_{e q} X_{2}(z)+k_{e q} X(z)-G(z)
$$

A similar relation can be deduced to compute the error in EL process in respect of $C_{e q}$ and $K_{e q}$. Using the basic procedure of the EL approach the solution of the nonlinear system is approximated. Equivalent parameters should be selected such that the error would be as small as possible by finding the mean square least error between the original equation and equivalent one [21].

$$
e^{2}=\left[c_{e q} X_{2}(z)+k_{e q} X(z)-G(z)\right]^{2}
$$

To determine the unknown coefficients from equation (11), the expected value of the error, $E\left[e^{2}\right]$, is derived and its derivations in respect of $c_{e q}$ and $k_{e q}$ are written. It should be noted that the expected value of the error has been considered for having generality. This is true where the error to be assumed as a random variable. The coefficients $c_{e q}$ and $k_{e q}$ are determined as follows:

$$
\frac{\partial E\left[e^{2}\right]}{\partial c_{e q}}=\frac{\partial E\left[e^{2}\right]}{\partial k_{e q}}=0
$$

Noting $E[x \dot{x}]=0$ due to displacement and the velocity are uncorrelated and by using BP transform properties from section (2), the Eq. (12) leads to the following equations:

$$
c_{e q}=\frac{\partial E\left[\left(\frac{2}{h} \frac{1-z^{-1}}{1+z^{-1}}\right) X(z) G(z)\right]}{E\left[\left(\frac{2}{h} \frac{1-z^{-1}}{1+z^{-1}}\right) X(z)\right]^{2}}
$$

and

$$
k_{e q}=\frac{\partial E[X(z) G(z)]}{E[X(z)]^{2}}
$$

In practical applications of involving numerical algorithms all parameters are computing at discrete grid points. In discrete domain the minimization conditions are:

$$
\frac{\partial}{\partial c_{e q}} \sum_{i=0}^{T} E\left[e^{2}\right]=0
$$


and

$$
\frac{\partial}{\partial k_{e q}} \sum_{i=0}^{T} E\left[e^{2}\right]=0
$$

In above equations, $T$ is the final time-instants in simulation process.

By solving equations (15) and (16) equivalent coefficients of the system can be determined as follows. Here, we found two expressions to calculate $c_{e q}$ and $k_{e q}$ using BP transform. Similar equations can be defined to find $C_{e q}$ and $K_{e q}$ according to EL approach.

$$
c_{e q}=\frac{\sum_{i=0}^{T}\left[\left(\frac{2}{h} \frac{1-z^{-1}}{1+z^{-1}}\right) x_{i} g_{i} z^{-i}\right]}{\sum_{i=0}^{T}\left[\left(\frac{2}{h} \frac{1-z^{-1}}{1+z^{-1}}\right) x_{i} z^{-i}\right]^{2}}
$$

and

$$
k_{e q}=\frac{\sum_{i=0}^{T} x_{i} g_{i} z^{-i}}{\sum_{i=0}^{T}\left(x_{i} z^{-i}\right)^{2}}
$$

For the summation (18) and (19) to converge, it is required that $\left|\frac{1}{z^{i}}\right|<1$ and $\left(1+z^{-1}\right)<\left(1-z^{-1}\right)$. We get $z>1$ and $z>0$ as convergence conditions. Therefore, the series do converge when $z$ larger than 1 .

The traditional linearization of the dynamic equation (7) leads to the following equation with $C_{e q}$ and $K_{e q}$ (in upper case letters), equivalent damping and stiffness coefficients to be determined by EL and replacement of the nonlinear terms of the main equation.

$$
m \ddot{x}(t)+C_{e q} \dot{x}(t)+K_{e q} x(t)=f(t)
$$

Again the replacement of a nonlinear system by a linear system is in some probabilistic sense and it will yield the difference or error. The error defined as

$$
e=g(x, \dot{x})-C_{e q} \dot{x}(t)-K_{e q} x(t)
$$

Minimizing the mean square value of $e$ is the criterion used here, i.e.

$$
e^{2}=E\left[\left(g(x, \dot{x})-C_{e q} \dot{x}(t)-K_{e q} x(t)\right)^{2}\right]
$$

The coefficient $C_{e q}$ and $K_{e q}$ are determined by the following equation: 


$$
\frac{\partial E\left[e^{2}\right]}{\partial c_{e q}}=\frac{\partial E\left[e^{2}\right]}{\partial k_{e q}}=0
$$

And we have

$$
C_{e q}=\frac{E[\dot{x} g(x, \dot{x})]}{E\left[\dot{x}^{2}\right]}
$$

and

$$
K_{e q}=\frac{E[x g(x, \dot{x})]}{E\left[x^{2}\right]}
$$

For more details see [1].

\section{Results}

To implement the proposed methodology for finding equivalent damping and stiffness coefficients of a system we consider a nonlinear SDOF system as shown in figure 1. Nonlinearity of system is modeled with nonlinear stiffness. The equation for a famous nonlinear oscillator, the Duffing equation is studied. The Duffing equation with nonlinear stiffness subjected to external signal $f(t)$ is written as follows.

$$
m \ddot{x}(t)+c \dot{x}(t)+g(x)=f(t)
$$

In which $m$ is the mass, $c$ is the damping coefficient, $f(t)$ is the external excitation and $x(t)$ is the displacement response of the system. The term, $g(x)$, is the nonlinear restoring force that would depend on displacement response defined as follows [22]:

$$
g(x)=k x+\alpha k_{3} x^{3}
$$

In the above equation $\alpha$ is considered additionally as non-linear factor that reflects the rate of nonlinearity in the nonlinear system.

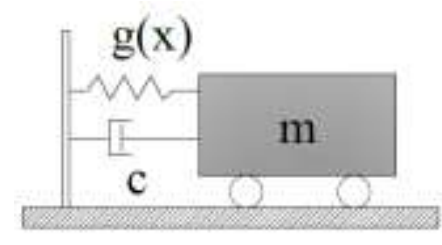

Fig. 1. SDOF nonlinear system

For instance, in an example system the dynamic parameters were chosen as follows, $m=2 \mathrm{~kg}, c=20 \mathrm{NSec} / \mathrm{m}, k=10^{4} \mathrm{~N} / \mathrm{m}$ and $k_{3}=3.8 \times 10^{9} \mathrm{~N} / \mathrm{m}$. The system was excited by two signals. The first test signal was the sine excitation which contains three different frequencies as $f(t)=\sin (2 \mathrm{t})+6 \sin (4 \mathrm{t})+4 \sin (6 \mathrm{t})$. The second one was a chirp signal in 
which the frequency increases linearly with time. In the linear chirp the frequency varies from $0.1 \mathrm{~Hz}$ to $1.5 \mathrm{~Hz}$ with a sampling period of 0.01 . It should be noted that the nonlinear Duffing system subjected to two stationary excitations. Using equation (17) and (18) for the stationary excitation may make some confliction. For the cases in which excitation is not stochastic the computed error is not random variable. It is obvious that in such cases expected value of error is replaced with sum of square errors and derivation of it in respect to unknown parameters is performed. The equivalent damping and stiffness coefficients of the Duffing system were calculated based on BP transform and EL method. To assess the effect of the rate of nonlinearity on the computed response of system, four different amount of nonlinear stiffness were considered through defining various $\alpha$. The values of 1.0, 1.05, 1.1 1nd 1.2 were considered. Figures 2 and 3 illustrate the displacement response of the linearized system using both proposed and traditional EL methods.

As illustrated in figures 2 and 3, three curves are plotted in each figures. a) The response of the linear system for the system in which $\mathrm{K}_{3}=0$. This one is illustrated only to show the disparity of the linearized system from linear response in respect of nonlinearity rate. It named "linear system" in figures. b) The response of nonlinear system linearized by the proposed method. c) The response of nonlinear system linearized by EL method.
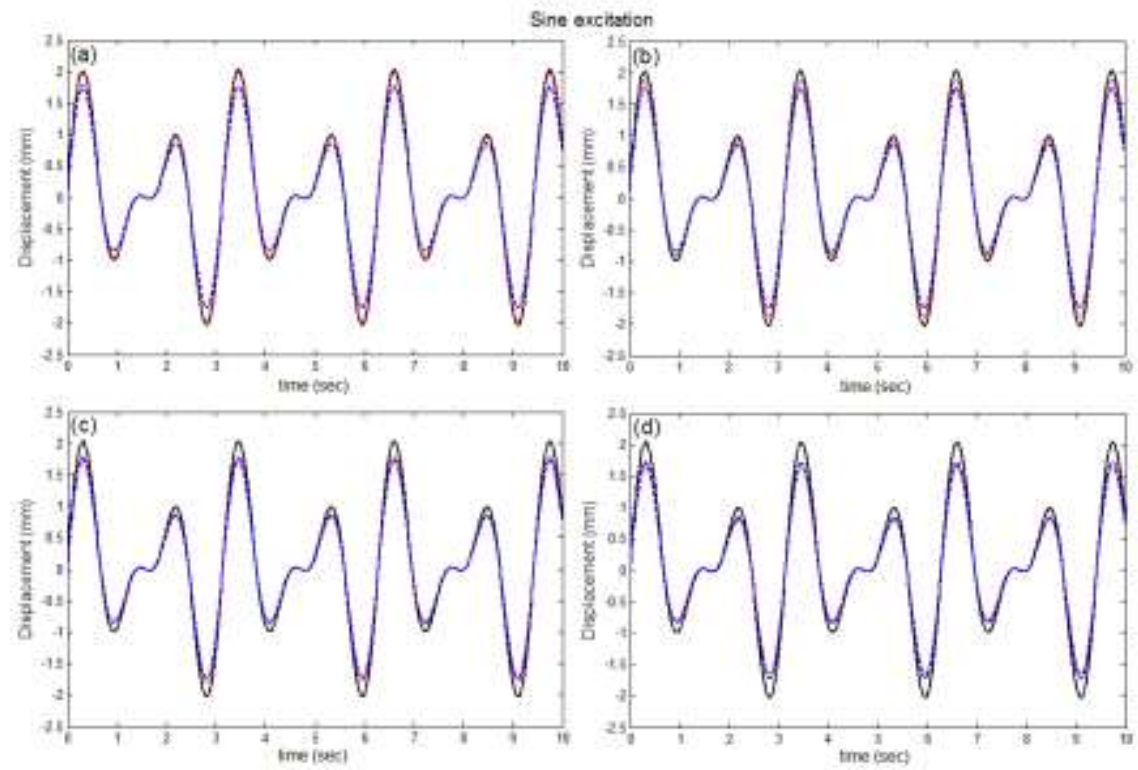

Fig. 2. Displacement response of linearized system; linear system (solid line), linearized system by proposed method (dotted line), linearized system by traditional EL method (dashed line): (a) $\alpha=1.0$ (b) $\alpha=1.05$ (c) $\alpha=1.1$ (d) $\alpha=1.2$.

The obtained results on nonlinear SDOF system reveal that the proposed linearization method based on BP transform can be a promising tool in linearizing nonlinear systems. Comparing the results shows that the difference between the responses obtained from two methods are very close to each other. The disparity of displacement response of linearized system based on BP transform from in respect to the response of linear system is plotted in figure 4 for values of $\alpha$. 

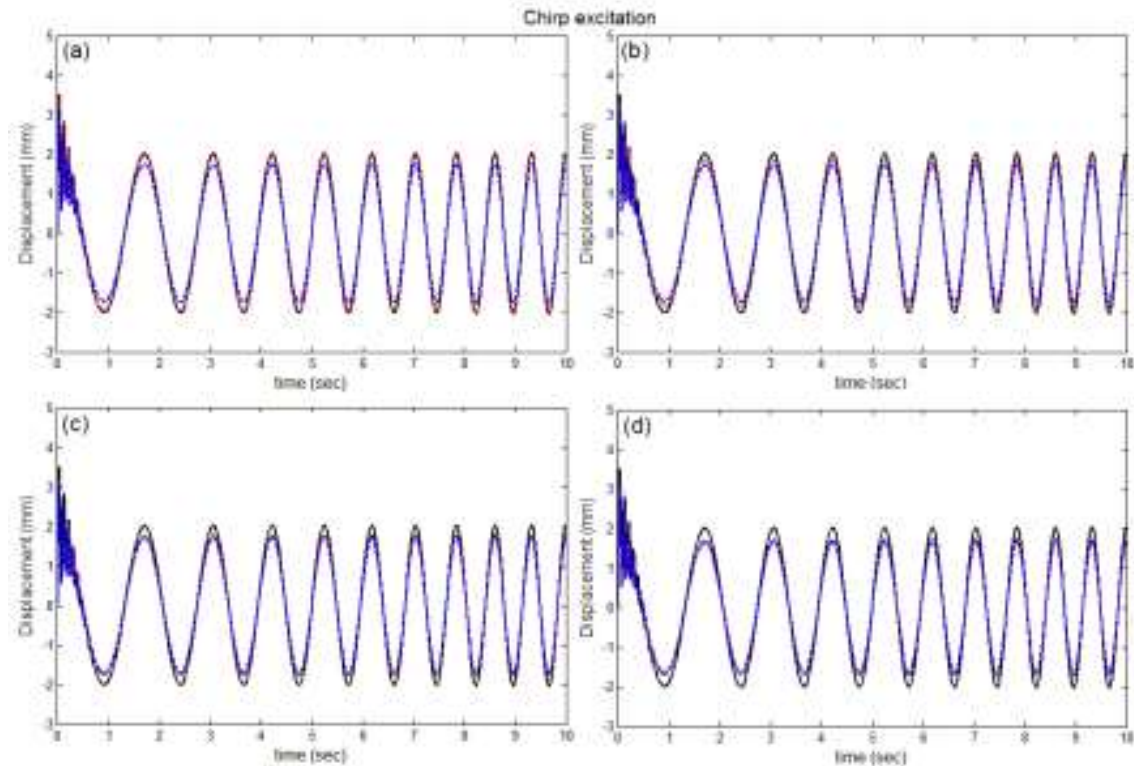

Fig. 3. Displacement response of linearized system; linear system (solid line), linearized system by proposed method (dotted line), linearized system by traditional EL method (dashed line): (a) $\alpha=1.0$ (b) $\alpha=1.05$ (c) $\alpha=1.1$ (d) $\alpha=1.2$.
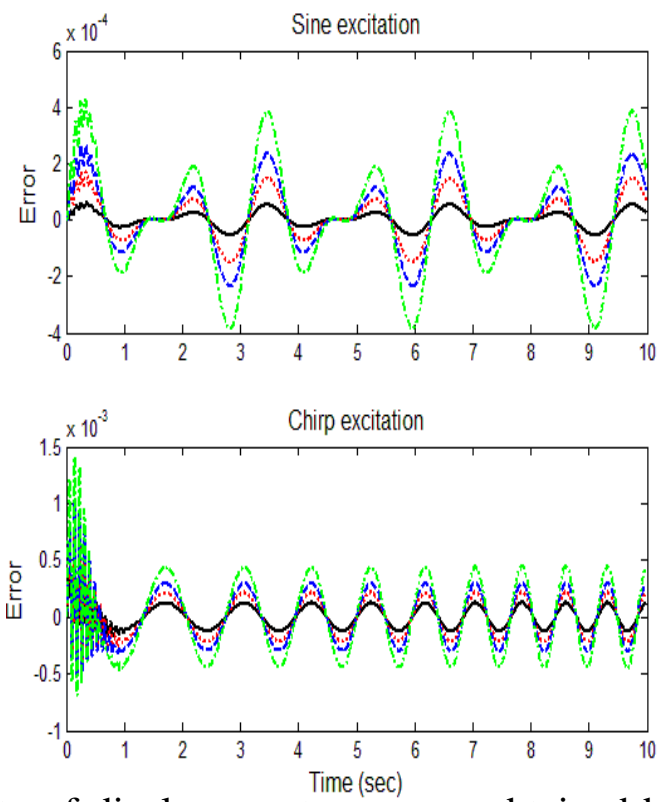

Fig. 4. The disparity of displacement response obtained based on the proposed method in respect to linear response: Displacement errors; $\alpha=1.0$. (solid line), $\alpha$ $=1.05$. (dotted line), $\alpha=1.1$. (dashed line), $\alpha=1.2$. (dash-dotted line).

In the field of structural dynamics, one of the most widely-used method of visualizing the dynamic properties of a system is to build the frequency response function (FRF). The FRF summarizes most of the necessary information such as resonances, anti-resonances, modal density and phase to specify the dynamics of a structure. In addition, the FRF can 
rapidly provide an indication of whether a system is linear or nonlinear. Figure 5 presents a comparison of FRF of the nonlinear system linearized by two methods.
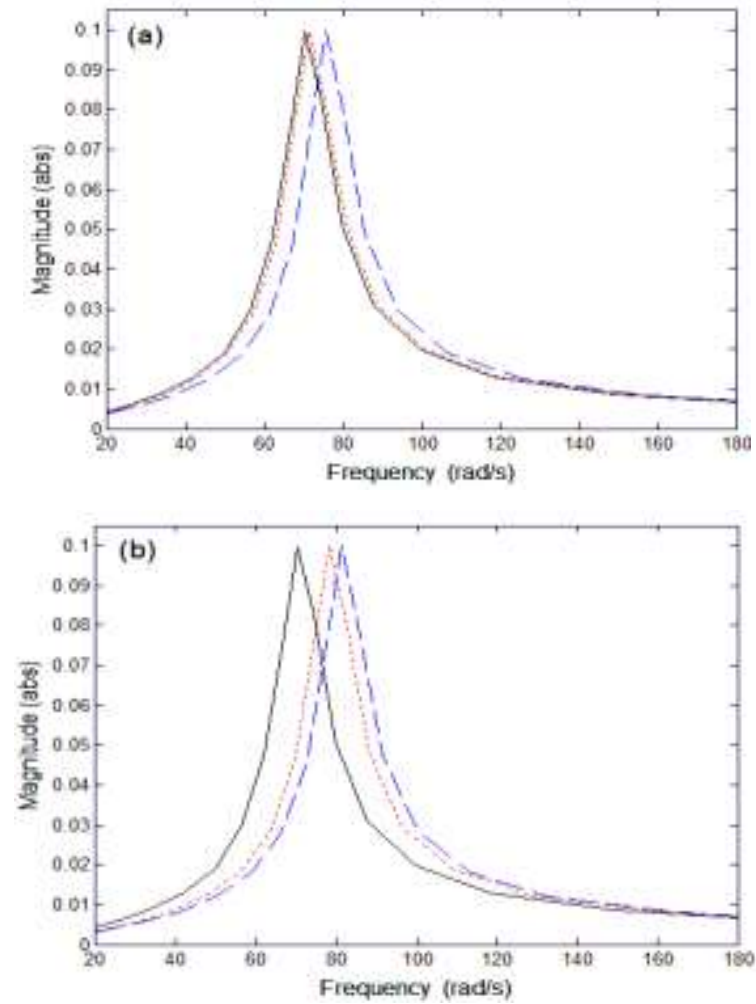

Fig. 5. Linearized FRF of system; linear system (solid line), linearized system by proposed method (dotted line), linearized system by traditional EL method (dashed line); (a) $\alpha=1.0$. and (b) $\alpha=1.2$.

\section{Conclusion}

This paper deals with linearization of a nonlinear SDOF system. The proposed method based on BP transform compared with traditional EL approach. In order to investigate the performance of the proposed linearization method, the Duffing oscillator subjected to stationary signals and the feasibility of the proposed method is demonstrated. The results showed that the proposed method can be suggested as a technique that simplifies solving complicated nonlinear dynamic equations. Comparisons between the results obtained based on two approaches reveal that for any rate of nonlinearity in nonlinear system, the displacement response of the proposed method is well-approximated and very close to those one obtained based on the traditional EL method. The frequency response functions of linear and linearized system have also determined. It is observed that the approximated nonlinear system by proposed method is well-behaved than the one approximated by traditional EL approach.

\section{REFERENCES}

[1] Worden K and Tomlinson GR 2001. Nonlinearity in structural dynamics. IOP Publishing Ltd, London.

[2] Liu T, Zordan T, Briseghella B and Zhang O. 2014. Evaluation of equivalent linearization analysis methods for seismically isolated buildings characterized by SDOF systems. Engineering Structutres, 59: 619-634. 
[3] Socha L. 2008. Linearization Methods for Stochastic Dynamic Systems. Springer, Berlin.

[4] Liu L, Thomas JP, Dowell EH, Attar P and Hall KC. 2006. A comparison of classical and high dimensional harmonic balance approaches for a Duffing oscillator. Journal of Computational Physics, 215: 298-320.

[5] Yue XK, Dai HH and Liu CS. 2014. Optimal scale polynomial interpolation technique for obtaining periodic solutions to the Duffing oscillator. Nonlinear Dynamics, 77: 1455-1468.

[6] Isidori A. 1989. Nonlinear control systems- An introduction. Springer, New York.

[7] Fuh CC and Wang MC. 2011. A Combined Input-state Feedback Linearization Scheme and Independent Component Analysis Filter for the Control of Chaotic Systems with Significant Measurement Noise. Journal of Vibration and Control, 17: 215-221.

[8] Deutscher J and Schmid CA. 2006. State space embedding approach to approximate feedback linearization of single input nonlinear control systems. International Journal of Robust and Nonlinear Control, 16: 421-440.

[9] Slotine JJ and Li W. 1991. Applied nonlinear control. Prentice-Hall, New Jersey.

[10] Caughey TK. 1963. Equivalent linearization techniques. Journal of the Acoustical Society of America, 35: 1706-1711.

[11] Jalali H. 2014. An alternative linearization approach applicable to hysteretic systems. Communication of Nonlinear Science and Numerical Simulation, 19: 245-257.

[12] Jiang WA and Chen LQ. 2014. An equivalent linearization technique for nonlinear piezoelectric energy harvesters under Gaussian white noise. Communication of Nonlinear Science and Numerical Simulation, 19: 2897-2904.

[13] Poularikas AD. 2010. Transforms and applications primer for engineers with examples and MATLAB. Taylor \& Francis Group, Boca Raton: CRC Press.

[14] Harmuth P.1969. Application of Walsh functions in communication. IEEE Spectrum 6: 82-91.

[15] PurnachandraRao V and RanganathaRao K. 1979. Optimal feedback control via block pulse function. IEEE Transanctions on automatic control, 24: 372-374.

[16]Sannuti P. 1977. Analysis and synthesis of dynamic systems via block pulse functions. Proc. IEEE 124: 569-571.

[17] Ghaffarzadeh H and Younespour A. 2014. Active tendons control of structures using block pulse functions. Structural Control and Health Monitoring, 21(12): 1453-64.

[18] Younespour A and Ghaffarzadeh H. 2014. Structural active vibration control using active mass damper by block pulse functions. Journal of Vibration and Control, DOI: $10.1177 / 1077546313519285$.

[19] Jiang ZH and Schaufelberger W. 1992. Block Pulse Functions and Their Applications in Control Systems. Springer, Berlin.

[20] Duffy DG. 2010. Advanced engineering mathematics with MATLAB. Taylor \& Francis Group, Boca Raton: CRC Press.

[21] Crassidis JL and Junkins JL. 2012. Optimal estimation of dynamic systems. Taylor \& Francis Group, Boca Raton: CRC Press.

[22] Dai HH, Yue XK and Yuan JP. 2013. A time domain collocation method for obtaining the third super harmonic solutions to the Duffing oscillator. Nonlinear Dynamics, 73: 593-609. 